\title{
Wire or Hook Traction for Reducing Zygomatic Fracture
}

\author{
Hee Chang Ahn', \\ Dong Hyun Youn ${ }^{1}$, \\ Matthew Seung Suk $\mathrm{Choi}^{2}$, \\ Jung-Woo Chang ${ }^{2}$, \\ Jang Hyun Lee ${ }^{2}$ \\ ${ }^{1}$ Department of Plastic and Reconstructive \\ Surgery, Hanyang University Medical Center, \\ Hanyang University College of Medicine, Seoul; \\ ${ }^{2}$ Department of Plastic and Reconstructive \\ Surgery, Hanyang University Guri Hospital, \\ Hanyang University College of Medicine, Guri, \\ Korea
}

No potential conflict of interest relevant to this article was reported.
Background: Variable methods have been introduced for reduction of the zygomatic fractures. The Dingman elevator is used widely to reduce these fractures but is inappropriate in certain types of fractures which require atypical traction vectors. We introduce and examine an alternate method of reducing zygomatic fractures using wire and hook traction. Methods: A retrospective study was performed for all zygomatic fracture patients admitted between 2008 and 2014. Medially rotated fractures were reduced by using a wire looped through an intermaxillary screw secured on the medial side of the zygoma. Laterally rotated fractures were reduced using a hook introduced through an infrazygomatic skin incision.

Results: No accidental bleeding or incomplete reduction was observed in any of the cases. Postoperative imaging demonstrated proper reduction immediately after the operation. Follow-up computed tomography study at 1 month after operation also demonstrated proper reduction and healthy union across the previous site of fracture.

Conclusion: The hook and wire method allowed precise application of traction forces across zygomatic fractures. The fractured bone fragment could be pulled in the direction precisely opposite to the vector of impact at the time of trauma. Soft tissue damage due to dissection was minimized. In particular, this method was effective in reducing rotated bone fragments and can be an alternative option to using the zygoma elevator.

Keywords: Facial bones / Fracture fixation / Zygoma

\section{INTRODUCTION}

The zygoma is a facial bone with a complex quadripod shape. It articulates with the frontal bone, temporal bone, maxilla, and sphenoid bone, and serves as the main bridge amongst these bones [1]. In addition, the bone provides anchor points for masseter, temporalis, and zygomaticus major and minor muscles [2]. The protruding malar eminence gives the face its three-dimensional appearance, and is a cause for the zygoma being a frequent site of facial fractures. The complex structure of zygoma

Correspondence: Jang Hyun Lee

Department of Plastic and Reconstructive Surgery, Hanyang University Guri Hospital, Hanyang University College of Medicine, 153 Gyeongchun-ro, Guri 11923, Korea

E-mail: jhlee1999@hanmail.net

Received June 12, 2015 / Revised October 23, 2015 / Accepted November 1, 2015 and narrow surgical field make for technically challenging operation. Improperly reduced zygomatic fracture may result in facial asymmetry, hypoesthesia, and mastication disability [1-3].

The zygoma must be sufficiently exposed for effective surgery. The frontozygomatic suture or arch can be accessed via the Gillies approach, lateral eyebrow approach, or coronal approach. As for the zygomaticomaxillary suture, it can be visualized via the subciliary approach or upper gingivobuccal approach [2]. While sufficient for visualization the fracture line, all of these approaches are remote from the fractures themselves, and the surgeon must attempt to reduce and fixate the fractures through a soft-tissue tunnel. In such operative contexts, the space may not be enough for instrumentation of the fracture site. In particular, traditional methods of zygoma reduction do not work well for depressed zygoma and for those fractures with soft tissue caught in-between. Forced reduction is most likely to be unsuccessful and increases the risk of hemorrhage 
and postoperative complication. In this study, we introduce a method of reducing zygomatic fracture using wire and hook traction and evaluate outcomes following the use of this method.

\section{METHODS}

A single-institutional retrospective study was performed for all patients who were admitted with the diagnosis of zygomatic fractures between 2008 and 2014. Of these, patients were excluded if the zygomatic fracture was simple arch fractures or bilateral zygomatic fractures. Patients were also excluded for comminuted fractures with segmented bones. The fracture type was classified according to the Knight and North [4] classification based on computed tomography (CT) images obtained before surgery.

\section{Surgical technique}

Upon general induction and surgical preparation, the fracture site was approached via the upper gingivobuccal sulcus access. The subciliary approach was utilized to identify fractures of the infraorbital rim. When necessary, the lateral eyebrow approach was used to sufficiently expose the frontozygomatic suture. According to the fracture pattern assessed before surgery, both of the following methods or one of them alone was performed.

\section{Wire reduction through screw fixation}

The wire method is useful for Group IV (Depression with medial rotation) fractures, where the bone segment is difficult to extract from the maxilla wall. With proper exposure of the fractured site, the bone is predrilled and the intermaxillary fixation screw is screwed into place. A 24-gauge wire is threaded through the hole in the head of this screw, and the fracture is reduced by pulling with the wire holder (Fig. 1). Once the bone is properly reduced, the wire and screw can be removed, and the remaining hole can be used for subsequent plate fixation across the fracture site.

\section{Percutaneous hook reduction}

This method is useful for Group V (depression with lateral rotation) fractures, where the zygoma is twisted laterally owing to the impac- tion. A 2-mm transverse skin incision is made at the inferior border of zygoma, and the hook is introduced through this access. With the hook securely anchored into the bone, the fractured zygoma is rotated anteromedially (Fig. 2). This approach is safe because the instrument does not have to traverse anatomically risky areas. Because the bone is thick, there is little risk of iatrogenic fracture. The skin incision can be closed with a single interrupted suture.

The reduced bones were fixed with plates and screws. The operative field was evaluated for hemostasis, and skin and mucosal wounds closed. Postoperative radiograph was taken on the day of operation. Patients were discharged in 1 week, and outpatient CT evaluation was performed at 1 month.

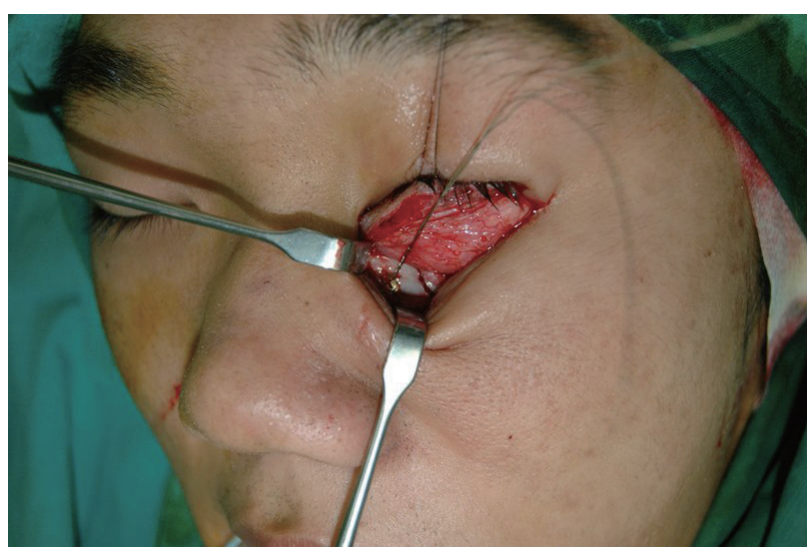

Fig. 1. Wire reduction through screw fixation. A 24-gauge wire is threaded through the hole in the head of this screw, and the fracture is reduced by pulling with the wire holder. The bone can be reduced by pulling on the wire holder.

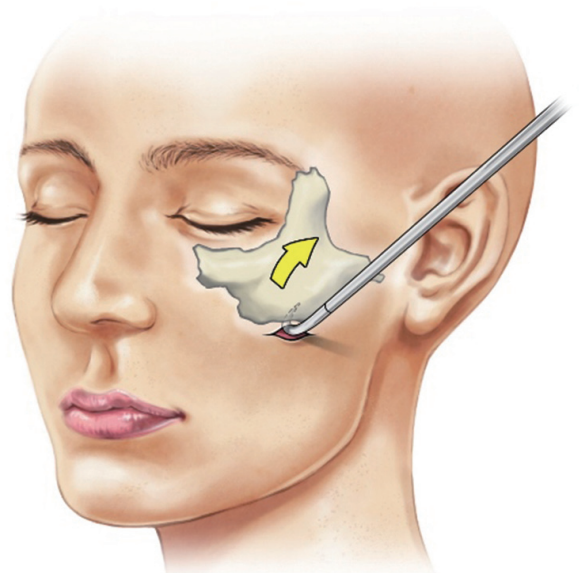

Fig. 2. Percutaneous hook reduction. The bone hook is inserted through the skin incision along the inferior border of zygoma. The hook is anchored to the bone, and the fracture is reduced with lateral traction. 


\section{RESULTS}

The review initially identified 171 patients who were admitted for zygomatic fracture, from which 31 patients were excluded according to the criteria (i.e., simple arch fracture, bilateral fractures, or comminuted fractures). The remaining 140 patients consisted of 119 male and 21 female patients. Most of the patients had been injured in a motor vehicle collision, followed by falls. Simultaneous facial bone fractures were present in 28 cases with orbital floor fracture being the most common, followed by nasal bone fracture (Table 1). Fracture patterns were divided according to the Knight and North classification, and the most frequent fracture class was Group V, followed by Groups I, IV, and III. By fracture type, both of the described methods or one of them alone was used in operation (Table 2). None of the patients experienced sudden intraopera-

Table 1. Summary of patient characteristics

\begin{tabular}{lc}
\hline Characteristic & Patients \\
\hline Sex & \\
Male & 119 \\
Female & 21 \\
\hline Age (yr) & \\
Mean (range) & $40(11-80)$ \\
\hline Affected side & \\
Right & 65 \\
Left & 75 \\
\hline Cause of injury & \\
Traffic accident & 45 \\
Slip down & 41 \\
Assault & 17 \\
Fall & 16 \\
Others & 21 \\
\hline Associated fracture & \\
Orbital floor & 13 \\
Nasal bone & 8 \\
Mandible & 6 \\
Others & 5 \\
\hline
\end{tabular}

Table 2. Reduction technique used in each type of fracture

\begin{tabular}{lcl} 
Type of fracture & Patients & Tools \\
\hline I (linear) & 35 & None \\
\hline III (depression without rotation) & 32 & Wire or hook reduction \\
\hline IV (depression with medial rotation) & 33 & Wire reduction \\
\hline V (depression with lateral rotation) & 40 & Hook reduction \\
\hline
\end{tabular}

Type II fractures were excluded in this study.

a) Knight and North classification. tive bleeding or incomplete reduction. Immediate postoperative radiographic studies were also satisfactory in all patients. Most patients were discharged within 1 week of operation. Follow-up CT exam at 1 month demonstrated no displacement or mal-union.

\section{DISCUSSION}

Continual efforts in safety regulations and education has resulted in measurable difference in the proportion of facial bone fractures caused by road traffic accidents. Despite these public health efforts, facial bone fractures still occur frequently [3]. In particular, a large percentage of midface fractures are zygomatic fractures $[5,6]$.

Zygomatic fractures are caused by high-energy impact, which can cause surrounding bones to break and become depressed and/or displaced. If displaced, zygomatic fractures should be reduced and fixed within the 2 weeks, and failure to address the fracture within this period can result in functional and aesthetic problems, such as enopthalmos and diplopia [1,6]. Properly timed and executed reduction is all the more important because the masseter muscle acts as an antagonist force against the zygomaticomaxillary buttress [7]. Unlike the infraorbital rim, the zygomaticomaxillary buttress is thick and plates on it do not tend to be palpable. Because of this, the bone allows firm fixation by plates [8]. The Dingman elevator is often used to reduce zygomatic fractures and currently provides the most effective method. Nevertheless, the Dingman elevator can be ineffective in rare types of zygomatic fractures.

Screws can be useful in reduction procedures because they can be pulled once they are fixed firmly in the bone. Matsuda et al. [9] had developed the hook screw for reduction of conventional fractures. Similarly, Kim et al. [10] and Moon et al. [11] introduced a method whereby a 2-mm intermaxillary fixation screw was used to anchor a wire across the fracture sites. Rao and Rao [12] demonstrated a method of reduction using a mono cortical screw and a self-holding screwdriver.

The titanium screws are available in variable diameters and lengths (1.5-2.0 $\mathrm{mm}$ and 6-8 $\mathrm{mm}$, respectively) and can be tightened into the cortical bone with just a drill. Depending on bone thickness, O'Regan and Devine [13] reported using 4-10 mm long 
screws via the same method. If fixed firmly and vertically in the bone, the screws do not fall out and convey enough strength. The attached wire can be pulled in the desired direction. This maneuver requires little space and can be performed through small incisions-thus reducing risk of soft tissue injury. The method is also applicable to reduction of the infraorbital rim or maxilla wall, as the small screws enables fine control of these thin bone fragments. The method is effective for Group IV (depression with medial rotation) fractures, which require a counterbalancing force to be applied to the anterior zygoma area (Fig. 3). The hole where the screw was inserted can also be used for plate fixation, which allows twopoint fixation of the infraorbital bone and zygomaticomaxillary buttress, and reduction by pulling on both at the same time [13]. However, screw fixation can only be performed in visible areas and is thus unsuitable for reduction of the lateral zygoma area.

For Group V fractures, a blind approach is unavoidable because the counterbalancing force needs to be applied to the lateral aspect of the zygoma. Despite the disadvantage belong to having a visible skin incision, the direct percutaneous approach can reduce risks that come with approaching the posterior portion of the zygoma. Previously, Park et al. [14] had reported external suspension
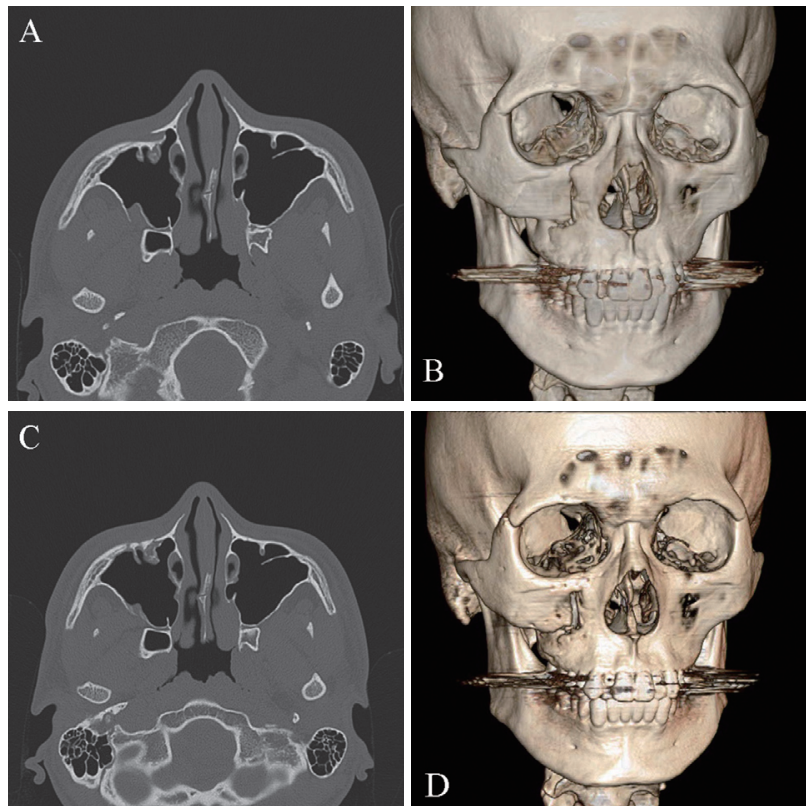

Fig. 3. Group IV zygomatic fracture. (A, B) Preoperative computed tomography views. (C, D) The depressed fragment was reduced using wire traction with screw and fixated with absorbable plates and screws. with a Kirchner wire after closed reduction and fixation using a Steinmann pin. Uda et al. [15] introduced closed reduction and internal fixation using a Carroll-Girard screw. All these reduction methods used a specific device via a slit incision over the malar eminence. Baek et al. [16] also performed reduction after fixation of a Carroll-Girard screw into the malar eminence via a lower eyelid incision. A bone hook can be used to reduce the fracture without damaging the bone by hooking through a relatively small incision at the inferior margin of zygoma (Fig. 4). This method is also allows anterior traction of a laterally rotated body [17].

Depressed fractures require a stabilizing force that is conveyed from inside to outside, but limited access to zygoma makes it difficult to apply sufficient force in this outward direction. These limitations make aggressive dissection necessary, and 2-point or 3-point fixation frequently require multiple approaches and extensive dissection to secure enough space [18]. Although accurate reduction is important, excessive incisions and soft tissue dissection can increase operating time and the risk of operative complications [19]. The use of wire and hook provides a secure method of reducing the zygomatic fracture while decreasing the amount of skin incision and soft tissue dissection.
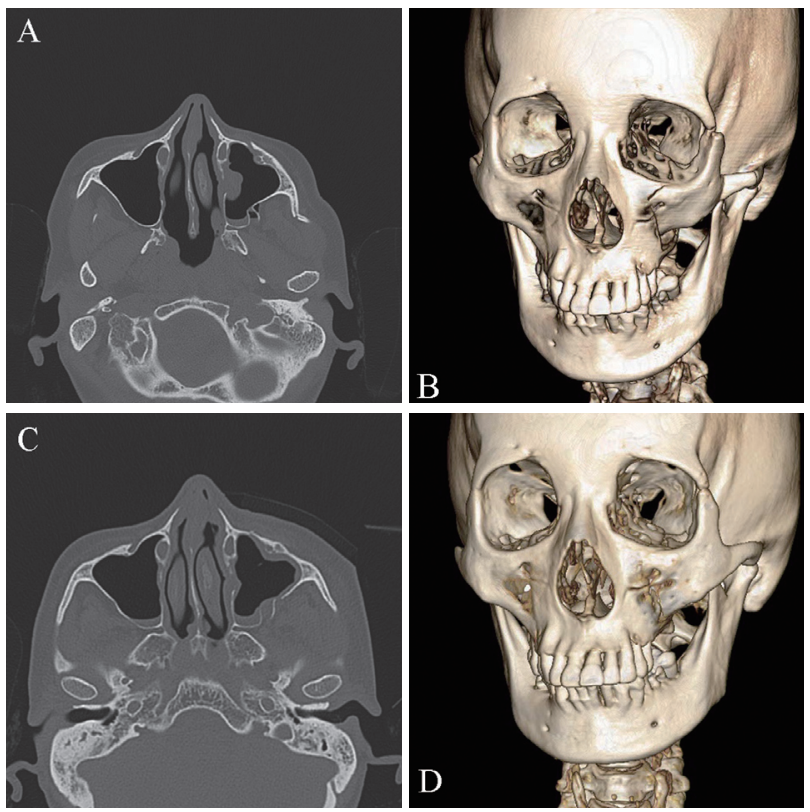

Fig. 4. A Group V zygomatic fracture. (A, B) Preoperative computed tomography images. (C, D) The depressed fragment was reduced using percutaneous hook and fixated with absorbable plates and screws. 
There are few limitations to the wire and hook method. First, it can be adjusted to simple fractures without comminution. The method is not appropriate for small bone fragments and depressed zygomatic arch. Second, hook traction has the potential to leave a scar at the skin incision site. Although it is only a slit incision, we have observed depressed or pigmented scar in some patients. Third, wire traction can only be performed if the target bone is solid enough to secure the screw.

Technically, the traction method using wire and hook has shown to be straightforward such that training residents often perform these complex reductions without difficulties at our institution. The wire and hook are common hardware items, which are all too readily available at any craniofacial center and are familiar to all surgeons working in the craniofacial field. Thus, the technique does not involve a novel operative maneuver and can be applied to a variety of zygomatic fractures.

\section{REFERENCES}

1. Kelley P, Hopper R, Gruss J. Evaluation and treatment of zygomatic fractures. Plast Reconstr Surg 2007;120(7 Suppl 2):5S-15S.

2. Ellstrom CL, Evans GR. Evidence-based medicine: zygoma fractures. Plast Reconstr Surg 2013;132:1649-57.

3. Meslemani D, Kellman RM. Zygomaticomaxillary complex fractures. Arch Facial Plast Surg 2012;14:62-6.

4. Knight JS, North JF. The classification of malar fractures: an analysis of displacement as a guide to treatment. Br J Plast Surg 1961;13:325-39.

5. Yamamoto K, Matsusue Y, Horita S, et al. Clinical analysis of midfacial fractures. Mater Sociomed 2014;26:21-5.

6. Salentijn EG, van den Bergh B, Forouzanfar T. A ten-year analysis of midfacial fractures. J Craniomaxillofac Surg 2013;41:630-6.
7. Hanemann M Jr, Simmons O, Jain S, et al. A comparison of combinations of titanium and resorbable plating systems for repair of isolated zygomatic fractures in the adult: a quantitative biomechanical study. Ann Plast Surg 2005;54:402-8.

8. Becelli R, Quarato D, Matarazzo G, et al. Esthetic positioning of rigid internal fixation in tripod zygomatic fractures: an innovative surgical technique. J Craniofac Surg 2009;20:724-5.

9. Matsuda K, Kubo T, Kawai K, et al. Use of hook screws in facial bone reduction. Plast Reconstr Surg 2005;115:1436-8.

10. Kim MG, Yoo RE, Chang H, et al. An intermaxillary fixation screw traction wire: an aid for facial bone fracture repair. Ann Plast Surg 2009;63:71-3.

11. Moon SH, Lee JH, Oh DY, et al. Reduction of zygomatic fracture segment with intermaxillary fixation screw. J Craniofac Surg 2012;23: 842-4.

12. Rao $S$, Rao S. Use of mono cortical screws as an aid to zygomatic complex fracture reduction. J Maxillofac Oral Surg 2015;14:126-7.

13. O'Regan B, Devine M. Screw-wire traction technique: aid to anatomical reduction of multi-segment mid-facial fractures. Br J Oral Maxillofac Surg 2013;51:459-60.

14. Park BY, Song SY, Yun IS, et al. First percutaneous reduction and next external suspension with Steinmann pin and Kirschner wire of isolated zygomatic fractures. J Craniofac Surg 2010;21:1060-5.

15. Uda H, Kamochi H, Sugawara Y, et al. The concept and method of closed reduction and internal fixation: a new approach for the treatment of simple zygoma fractures. Plast Reconstr Surg 2013;132:1231-40.

16. Baek JE, Chung CM, Hong IP. Reduction of zygomatic fractures using the carroll-girard T-bar screw. Arch Plast Surg 2012;39:556-60.

17. Ward Booth P, Hausamen JE, Schendel SA. Maxillofacial surgery. St. Louis, Mo.: Churchill Livingstone/Elsevier; 2007.

18. Czerwinski M, Martin M, Lee C. Quantitative comparison of open reduction and internal fixation versus the Gillies method in the treatment of orbitozygomatic complex fractures. Plast Reconstr Surg 2005;115:1848-54.

19. Sargent LA, Fernandez JG. Incidence and management of zygomatic fractures at a level I trauma center. Ann Plast Surg 2012;68:472-6. 